\title{
The influence of moisture on potassium bromide disks used in infrared spectrometry*
}

\author{
J. H. van der MaAs and A. Tolk \\ Analytical Chemical Laboratory \\ State University, Utrecht, The Netherlands
}

(Received 29 June 1961)

\begin{abstract}
When using the alkali-halide disk technique in infrared spectrometry we noticed that moisture from the atmosphere can penetrate into a $\mathrm{KBr}$ disk, causing recrystallization of the $\mathrm{KBr}$. A critical relative humidity was observed above which moisture is taken up. This value depends on the fineness and structure of the $\mathrm{KBr}$; for optical grade, handground $\mathrm{KBr}$ (Merck) it is about 70 per cent. "Wet" disks lose water if stored in an atmosphere of a lower humidity; replaced in the original atmosphere, they take up water again but to a lower extent.

In order to study the water sensitivity of $\mathrm{KBr}$ disks containing a sample, KSCN was used. $\mathrm{KSCN}$ has two $\mathrm{C}^{\prime} \equiv \mathrm{N}$ stretching bands dependent on the form in which it occurs: as a solution at $4.84 \mu$ and in the solid phase at $4.87 \mu$. The latter can be converted into the former by water uptake of the disk; on drying of the disk the band is shifted back to its original wavelength.

In the disk are small cracks through which condensed moisture and dissolved sample can be transported. This is demonstrated by the behaviour of KSCN. If a $\mathrm{KBr}$ disk, containing a single KSC.N crystal completely surrounded by carrier material, is exposed to a moist atmosphere the KSCN is transported through the whole disk.

The same transport, due to the presence of water in a disk, was observed with a number of similar inorganic salts. Nometimes the accompanying bandshifts could be eliminated by drying, sometimes they persisted, probably indicating that a solid solution has been formed. In the absence of water none of these phenomena were observed.

Interaction between sample and water can be demonstrated also with $\mathrm{D}_{2} \mathrm{O}$. In a moist $\mathrm{D}_{2} \mathrm{O}$ atmosphere active $\mathrm{H}$ atoms of sample molecules in a disk are substituted by $\mathrm{D}$ atoms. This phenomenon can be applied successfully in structure determination.
\end{abstract}

\section{Introduction}

THE KBr disk technique is a valuable sampling method in infrared spectrometry. A number of substances however, show or can show anomalous spectra if prepared according to this method (e.g. benzoic acid). If a disk containing benzoic acid in the anomalous form is exposed during some time to an atmosphere with a rather high relative humidity a complete conversion into the normal form of the acid takes place, as was found some time ago in our laboratory [1].

The work presented here deals with:

A. The water content of $\mathrm{KBr}$ disks.

B. Redistribution of sample material by penetrating water.

C. Conversions caused by water.

D. The use of a $\mathrm{D}_{2} \mathrm{O}$ atmosphere.

\section{Methods and materials}

In the experiments two kinds of $\mathrm{KBr}$ were used: Merck reagent grade and Merck optical

* This paper was presented at the Fifth European Congress on Molecular Spectroscopy, Amsterdam, 29 May $\rightarrow 3$ June 1961.

[1] A. Touk, Spectrochim. Acte 17, 511 (1961). 
grade resp. After grinding in an agate mortar, these materials were stored at about $130^{\circ} \mathrm{C}$ ready for use. The sample materials were reagent grade.

The atmospheres with distinct relative humidities were obtained with the help of saturated solutions of $\mathrm{LiCl}, \mathrm{K}_{2} \mathrm{CO}_{3}, \mathrm{NaBr}, \mathrm{NaCl}$ and $\mathrm{Na}_{2} \mathrm{~h}_{2} \mathrm{O}_{3}$ giving relative humidities of respective $\mathrm{y}_{2} 12$, $44,57,75$ and 78 per cent at room temperature according to RockLAND [2].

Different amounts of sample and always about $300 \mathrm{mg} \mathrm{KIBr}$ were mixed in an agate mortar and put into an evacuable die with splittable cone (internal diameter $13 \mathrm{~mm}$ ) according to Schiedt [3]. The mixture was evacuated for $3 \mathrm{~min}$ and then a pressure of 12 tons was applied for 3 min under maintenance of the vacuum.

The spectra were recorded with a Perkin-Flmer 21 spectrophotometer linear in wavelength. and equipped with a $\mathrm{NaCl}$ prism.

\section{Experiments, results and discussion}

\section{A. The water content of $\mathrm{KBr}$ disks}

We investigated the amount of water which is taken up by disks if they are stored in atmospheres with various relative humidities. The amount was calculated from the IR band at $2 \cdot 9 \mu$. using an extinction value of $1 \cdot 25$ per $\mathrm{mg}_{2} \mathrm{O}$ [4]. The results are given in Table 1 for two kinds of $\mathrm{KBr}$ and for $\mathrm{KBr}$ containing some KSCN.

Table 1. The water-content in $\mu \mathrm{g}$ per disk of about $300 \mathrm{mg} \mathrm{KBr}$. First number: instantly after pressing; Second number: after $96 \mathrm{hr}$ storage at the indicated relative humidity. For the experiments with $\mathrm{KSCN} 0.2 \mathrm{mg}$ of this salt was mixed with about $300 \mathrm{mg} \mathrm{KBr}$.

\begin{tabular}{|c|c|c|c|c|c|c|}
\hline \multirow{2}{*}{$\begin{array}{l}\text { Relative humidity } \\
\frac{\%}{12}\end{array}$} & \multicolumn{2}{|c|}{$\mathrm{KBr}$ optical } & \multicolumn{2}{|c|}{ KBr reagent } & \multicolumn{2}{|c|}{$\mathrm{KBr}+\mathrm{KSCN}$} \\
\hline & 7 & 7 & 10 & 14 & 20 & 30 \\
\hline 44 & 7 & 7 & 10 & 17 & 20 & 124 \\
\hline 57 & 3 & 7 & 10 & 20 & 23 & 309 \\
\hline 75 & 7 & 69 & 7 & 99 & 17 & 611 \\
\hline 78 & 7 & 295 & 10 & 325 & \multicolumn{2}{|c|}{ opaque } \\
\hline
\end{tabular}

As can be seen there is no great difference between optical and reagent grade KBr. At a relative humidity of 57 per cent or lower no water is taken up. At 75 per cent or higher the water uptake is significant $\left(0.007 \mathrm{~mol} \mathrm{H}_{2} \mathrm{O} / \mathrm{mol} \mathrm{KBr}\right)$. Since stable crystals of $\mathrm{KBr}$ absorb water only at a relative humidity of 84 per cent [5], we must conclude that the $\mathrm{KBr}$ in the disk, or part of it, is present in a less stable state.

Furthermore we observed that "wet" disks lose water if stored at lower relative humidities; replaced in the original atmosphere they take up water again but to a lower extent.

The $\mathrm{KBr}$ disks, containing some $\mathrm{KSCN}$ show a significant increase in water uptake. At 44 per cent it is quite considerable already. Since the critical relative

[2] L. B. Rocktand Anal. Chem. 32, 1375 (1960),

[3] U. Schrent, Z. Nuturforsch. 8b, 66 (1953).

[4] A. ToLx, Thesis, University Utrech (1959).

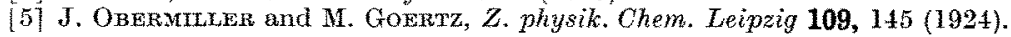


humidity for KSCN (pure) is about 47 per cent [5], the water uptake of $\mathrm{KBr}$ disks containing a solid sample, seems to be influenced by the properties of this sample.

B. Redistribution of sample material by penetrating water

For these experiments disks containing some KSCN were used. Dry $\mathrm{KBr}$ $(300 \mathrm{mg})$ and $\mathrm{KSCN}(0 \cdot 2 \mathrm{mg})$ were mixed carefully and directly after pressing a spectrum was run. A very small water band at $2.9 \mu$ was observed and a rather

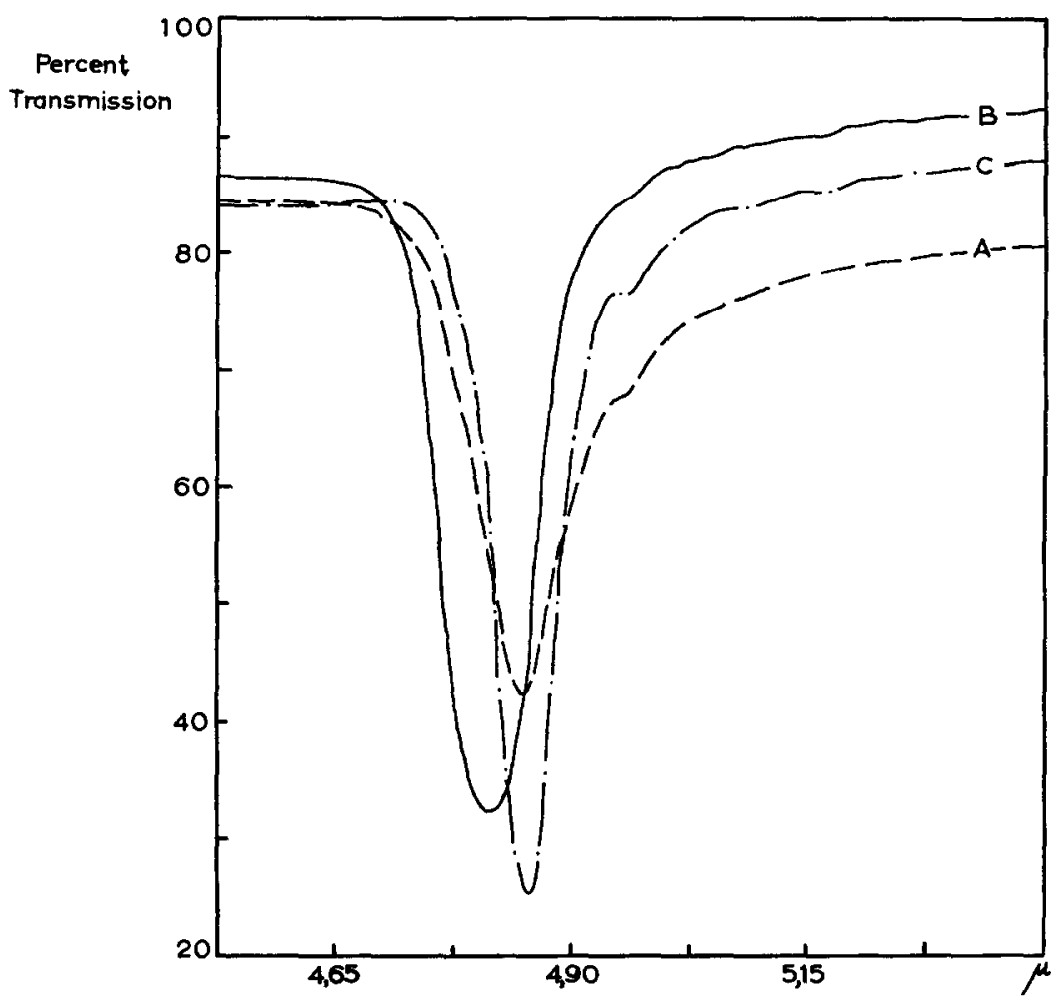

Fig. 1. The $\mathrm{C} \equiv \mathrm{N}$ stretching vibration of KSCN. Curve (a) After pressing.

(b) After storage at 57 per cent relative humidity. (c) After drying.

strong band at $4.87 \mu$ due to the $\mathrm{C} \equiv \mathrm{N}$ stretching vibration of KSCN in the solid state (Fig. 1, curve a).

After $3 \mathrm{hr}$ storage at a relative humidity of 57 per cent the spectrum showed a very strong waterband at $2.9 \mu$, while the maximum of the $\mathrm{C} \equiv \mathrm{N}$ stretching band was shifted to $4 \cdot 84 \mu$ (Fig. 1, curve b).

Drying of the wet disk above $\mathrm{P}_{2} \mathrm{O}_{5}$ in vacuo resultcd in a spectrum with little or no water absorption at $2.9 \mu$ and a $\mathrm{C} \equiv \mathrm{N}$ stretching band at $4.87 \mu$ which is even stronger then before the water treatment (Fig. I, curve c).*

Since a KSCN solution in water absorbs at $4.84 \mu[7]$ the KSCN in the wet

* Due to this sensitivity for moisture KSCN cannot be used as internal standard as proposed by WiberLex et al. [6].

[6] S. E. Wiberley, J. W. Sprague and J. E. Campbell, Anal. Chem. 29, 210 (1957).

[7] L. H. Jones, J. Chem. Phys. 25, 1069 (1956). 
disk is hydrated to a structure, resembling or even identical with, its structure in water solution. We therefore are inclined to assume the following mechanism: water from the surrounding atmosphere condenses and penetrates into the disk through small cracks. The KSCN particles are hydrated or even dissolved, and the $\mathrm{SCN}$ ions are transported through the whole disk, resulting in a more homogeneous distribution of the KSCN. On drying the water evaporates, while the KSCN crystallizes into smaller particles then before, resulting in a stronger absorption at $4 \cdot 87 \mu$. Perhaps it even forms a solid solution in $\mathrm{KBr}$.

Besides, the transport of the SCN ions by the penetrating water was demonstrated as follows: a small KSCN crystal was mounted excentrically in a disk in such a way that it was completely surrounded by $\mathrm{KBr}$. 'This disk did not show a KSCN spectrum because only the centre part of the disk is used for absorption measurements. Storage of the disk for some weeks in a dry atmosphere had no effect. After storage during $20 \mathrm{~min}$ in an atmosphere with a relative humidity of 57 per cent however, the $4.84 \mu$ band appeared. After storage for $1 \frac{1}{2} \mathrm{hr}$ this band was strong. A superficial study of the disk using the Perkin-Elmer micro-illuminator showed that the water and thiocyanate concentrations were high in the close vicinity of the KSCN particle. The concentrations decreased with increasing distance.

From another disk treated in the same way we determined after drying the thiocyanate concentration, again with the help of the micro-illuminator. along the $x$ - and $y$-axis. The results are given in arbitrary units in Fig. 2 , using the $4 \cdot 87 \mu$ band for calculations. They are in agreement with the expectations.

So we may conclude, that water from the surrounding atmosphere enters the disk where the crystal(s) is (are) embedded; it dissolves the sample and transports it through the whole disk.

We have found that potassium acetate, $\mathrm{KNO}_{2}, \mathrm{KNO}_{3}, \mathrm{NH}_{4} \mathrm{SCN}, \mathrm{KHF}_{2}$ and $\mathrm{NH}_{4} \mathrm{HF}_{2}$ are transported also (at relative humidity of 78 per cent), though there is a significant difference in lransport speed, presumably due to the difference in solubility, e.g. $\mathrm{NaHF}_{2}$ is transported very slowly and is indeed less soluble.

\section{Conversions caused by water}

Water proved to initiate conversion of a salt in a disk as was found by VRÁTNÝ [8] in the case of some nitrates.

We observed this phenomenon also with $\mathrm{NH}_{4} \mathrm{SCN}$ : a $\mathrm{KBr}$ disk containing some ammoniumthiocyanate remains unchanged if the disk is perfectly dry. After storage during 60 min at a relative humidity of 78 per cent and drying afterwards, the spectrum in the $10-11 \mu$ region indicates that the $\mathrm{NH}_{4} \mathrm{SCN}$ has been transformed: the bands at 10.5 and $10.7 \mu$ are shifted to 10.3 and $10.5 \mu$ respectively (Fig. 3, curve a and c). Recording the spectrum after $30 \mathrm{~min}$ without drying. shows that the conversion has taken place only partially. (Fig. 3. curve b).

This phenomenon can be due to either a conversion of ammonium into potassium thiocyanate, or to the formation of a solid solution in $\mathrm{KBr}$. In the last case

[8] F. VRÁtNÝ, J. Inorg. \& N'uclear Chem. 10, 328 (1959). 
we must assume that the spectrum of the SCN ions surrounded by $\mathrm{K}^{+}$and $\mathrm{Br}^{-}$ ions is the same as that of pure KSCN, because the spectrum of this salt is not shifted after ageing and drying, as we saw before.

If disks with respectively $\mathrm{K}, \mathrm{NH}_{4}$ and $\mathrm{NaHF}_{2}$ are exposed to a moist atmosphere (78 per cent) new bands are observed at 6.55 and $7.98 \mu$ in all cases, belonging to $\mathrm{HF}_{2}{ }^{-}$vibrations. KETELAAR et al. [9] ascribe these new bands to the formation of

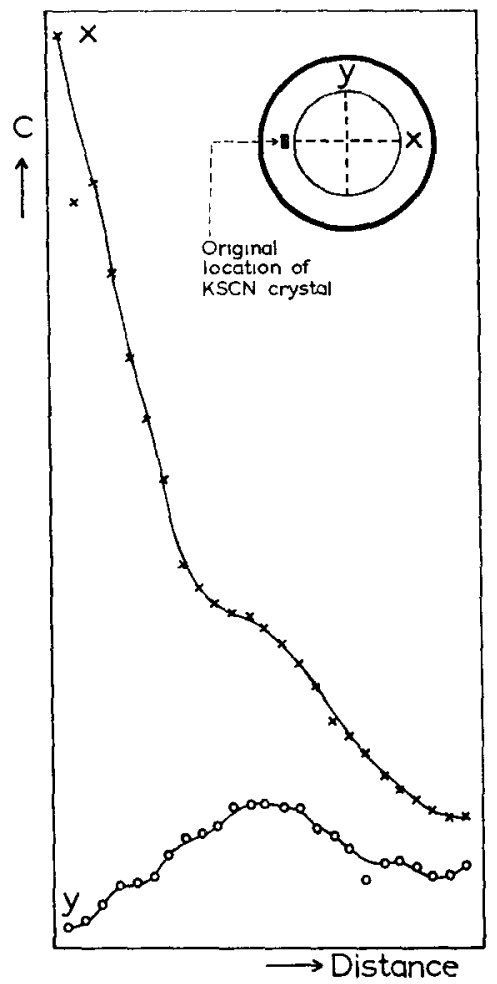

Fig. 2. The KSCN coneentration $C$ (in arbitrary units) in small volumes of a disk along the $x$ - and $y$ axis, as indicated in this figure.

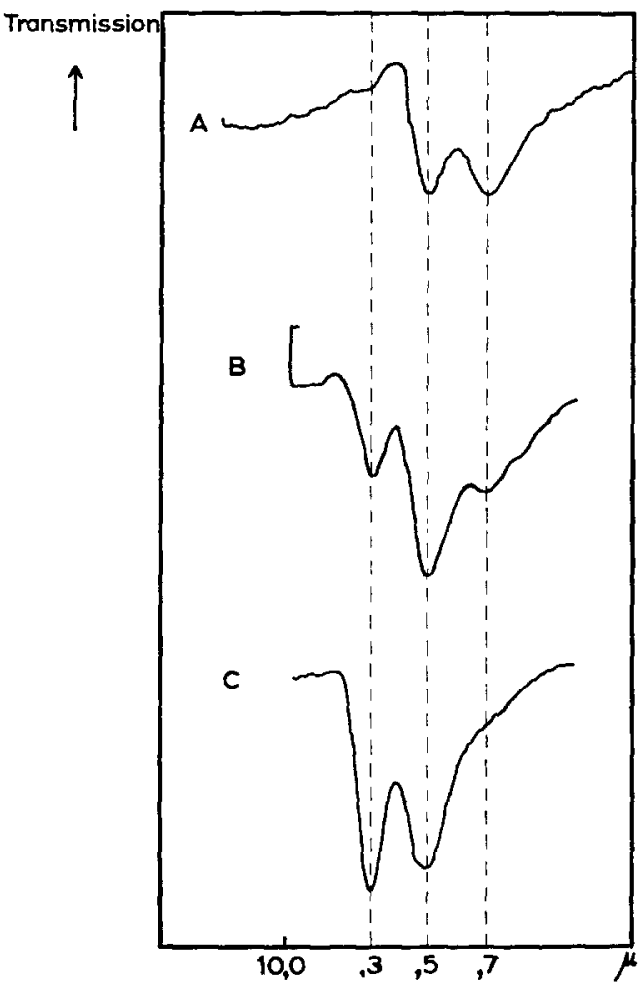

Fig. 3. The conversion of $\mathrm{NH}_{4} \mathrm{SCN}$ followed in the $10-11 \mu$ region. (a) Directly after pressing (no conversion). (b) After $30 \mathrm{~min}$ storage at a relative humidity of 78 per cent (partially converted). (c) After $60 \mathrm{~min}$ storage and drying (completely converted).

mixed crystals; the original vibrations of the $\mathrm{HF}_{2}$ ions correspond with bands on other places.

We have found that the shifts do not occur if water is absent, e.g. a disk containing $\mathrm{NH}_{4} \mathrm{HF}_{2}$ after storage for three months above $\mathrm{P}_{2} \mathrm{O}_{5}$ showed still the same spectrum as instantly after pressing. In a moist atmosphere however the original $\mathrm{HF}_{2}$ bands of the $\mathrm{K}$ and $\mathrm{NH}_{4}$ salt disappear completely during ageing; those of the $\mathrm{Na}$ salt nearly and after a longer ageing period. This is presumably due to the small transport speed as mentioned earlier. In our opinion it is probable that in all three cases solid solutions occur.

[9] J. A. A. KetelaAr, C. HaAs and J. van der Elsken, J. Chem. Phys. 24, 624 (1956). 
Comparing these results with those obtained with $\mathrm{NH}_{4} \mathrm{SCN}$ we are inclined to suppose that there too the formation of a solid solution is possible or even likely, though a definite judgement cannot be given yet.

Our over-all conclusion is that the presence of water is essential for the conversion of a sample in a $\mathrm{KBr}$ disk and that this water can be taken up easily from the surrounding atmosphere.

\section{The use of a $\mathrm{D}_{2} \mathrm{O}$ atmosphere}

For an application of the results discussed above deuteriumoxyde was used.

If a disk containing a sample is stored for a couple of hours in a moist $\mathrm{D}_{2} \mathrm{O}$ atmosphere (relative humidity 75 per cent), active $\mathrm{H}$ atoms of the sample molecules can be substituted more or less by $\mathrm{D}$ atoms from the penetrated $\mathrm{D}_{2} \mathrm{O}$. This will cause significant bandshifts, which can be applied in structure determination.

Such experiments were done with: benzoic, cinnamic, salicylic and phthalic acid and with resorcin, $\alpha$-naphthol, androsteron, urea and acetamide. In all these cases bandshifts have been observed indeed.

Acknowledgements--'The authors are indebted to Prof. Dr. Ir. J. SmITtenBert for the facilities to carry out this investigation and to Miss A. M. Wesseltus, who did most of the experimental work. The investigations have been performed under the auspices of the Netherlands Foundation for Chemical Research (S.O.N.) and with financial aid from the Netherlands Organisation for the Advancement of Pure Research (Z.W.O.) 